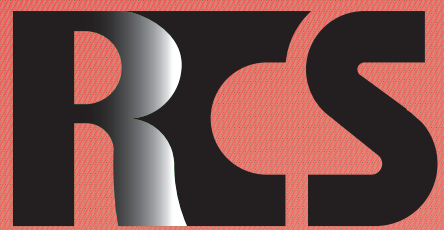

Depósito legal ppi $201502 Z U 4662$

Esta publicación científica en formato digital es continuidad de la revista impresa Depósito Legal: pp $197402 Z U 789$

- ISSN: 1315-9518 • ISSN-E: 2477-9431

Revista de Ciencias Sociales

Universidad del Zulia. Revista de la Facultad de Ciencias Económicas y Sociales Vol. XXVII. No. 2

Abril-Junio 2021

Esta publicación científica en formato digital es continuidad de la revista impresa Depósito Legal: pp $197402 Z$ Z789 ISSN: 1315-9518 

social: ¿Nueva cara de la responsabilidad social? conceptualización crítica desde la perspectiva universitaria. Revista de Ciencias Sociales (Ve),

\title{
Innovación social: ¿Nueva cara de la responsabilidad social? conceptualización crítica desde la perspectiva universitaria
}

\author{
Vargas-Merino, Jorge Alberto*
}

\section{Resumen}

Comprender la innovación social es una tarea compleja, pluriconceptual y dinámica. Se puede evidenciar el interés creciente en diferentes grupos, perspectivas y disciplinas, por desentrañar su distinción teórica y conceptual, puesto que existe una amplia variedad de definiciones que la califican de ambigua por su misma naturaleza multidisciplinar ¿Se está frente a una nueva concepción de la responsabilidad social? Por ello, este artículo busca analizar dilucidar y entender la complejidad de la definición de la innovación social y el rol que esta estaría forjándose desde la Universidad, mediante revisiones exploratorias que permitan sintetizar la evidencia existente y aplicando criterios de evaluación de la calidad de los documentos encontrados. Entre los resultados destaca que la gestión de las empresas no se condice con la misión y visión de las mismas, buscando sólo un incremento de su reputación con acciones paliativas. Se puede concluir, que innovación social es un término difuso, ambiguo y multivariado, sin embargo, existe consenso en cuanto a atención de necesidades sociales y ambientales no cubiertas y su contribución a mejorar la calidad de vida, desde un enfoque técnico así como aplicado, con soluciones proactivas y duraderas, demandando participación de la universidad desde su quehacer medular: La investigación.

Palabras clave: Innovación social; responsabilidad social; enfoque crítico; universidad; revisión teórica.

\footnotetext{
$<$ ? $>\quad$ "Doctorando en Ciencias Administrativas por la UNMSM-Perú. Magíster en Economía. Magíster en Administración. Licenciado en Administración. Docente Investigador en la Universidad Privada del Norte, Perú. E-mail: jorgealbvarmer5@gmail.com; jorge.merino@upn.pe (iD ORCID: https://orcid.org/0000-0002-3084-8403.
} 


\title{
Social Innovation: New Face of Social Responsibility? critical conceptualization from the university perspective
}

\begin{abstract}
Understanding social innovation is a complex, multi-conceptual and dynamic task. The growing interest in different groups, perspectives and disciplines can be evidenced, to unravel its theoretical and conceptual distinction, since there is a wide variety of definitions that qualify it as ambiguous due to its very multidisciplinary nature. social responsability? Therefore, this article seeks to analyze, elucidate and understand the complexity of the definition of social innovation and the role that this would be forging from the University, through exploratory reviews that allow synthesizing the existing evidence and applying criteria for evaluating the quality of the documents. found. Among the results, it stands out that the management of the companies is not consistent with their mission and vision, seeking only an increase in their reputation with palliative actions. It can be concluded that social innovation is a diffuse, ambiguous and multivariate term, however, there is consensus regarding the attention to unmet social and environmental needs and its contribution to improving the quality of life, from a technical as well as an applied approach, with proactive and lasting solutions, demanding the participation of the university from its core task: Research.
\end{abstract}

Keywords: Social innovation; social responsibility; critical approach; university; theoretical review.

\section{Introducción}

Hernández-Ascanio, Tirado-Valencia y Ariza-Montes (2016), expresan que, a pesar de ser un elemento terminológico en apogeo, la literatura científica en la actualidad carece de un acuerdo formal del concepto de innovación social. Se está frente a un criterio sumamente elástico, el cual puede ser expuesto desde variadas perspectivas disciplinarias y distintos ámbitos.

Esta acepción compleja del término se amplía con la existencia de innumerables estudios que coexisten en la sociedad, tales como Mesthene (1970); Chambon, David y Devevey (1982); Crozier y Friedberg (1993); Christensen, et al. (2006); Echevarría (2008); Abreu (2011); Mendes, et al. (2012); Conejero y Redondo (2016), quienes precisamente no hallan una definición estándar o única como en otros campos o áreas del conocimiento, o comparada con la innovación clásica, tampoco hay consenso respecto a su alcance ni a su aplicación; esto dificulta que se instauren patrones sistematizados que posibiliten la investigación científica exhaustiva.

Hernández-Ascanio, et al. (2016), hacen referencia que ante un gran conjunto de problemas de índole social y medioambiental que aquejan constantemente, y que son parte de una sociedad donde sus principales actores se muestran indolentes y poco accesibles a emprender soluciones que den como resultado impactos positivos perdurables. Surge la necesidad de iniciar trabajos que propicien resultados innovadores, a la par que representen atributos de tipo social.

En su mayoría los resultados presentados sobre los conflictos sociales son pasajeros y precarios. La Comisión Económica para América Latina y el Caribe (CEPAL), desde el 2004 viene investigando sobre la innovación social, hallando varios ejemplos de cómo los Estados no les dan respuesta efectiva a las necesidades de los individuos, puesto que suelen centrarse en procesos y métodos tradicionales; y con el paso del tiempo se observa una acentuación de las problemáticas en lugar de mitigarlas o eliminarlas (CEPAL, 
2011); por lo consiguiente, se busca la incorporación de innovadores, los cuales estén encausados en la creación del valor social, con estrategias que puedan ser replicables en diferentes escenarios y contextos, y a la vez sostenibles, con su correspondiente orientación propendida hacia la igualdad e inclusión social (Parada, Ganga y Rivera, 2017).

La época actual que se está viviendo, está impregnada de diversos conflictos económicos, sociales y ambientales que entorpecen el progreso de las sociedades, indistinto del oficio en el cual se accione, se afronta enormes desafíos, y ya no se trata de lo que se debería hacer, sino de lo que se debe hacer en donde se interactúa. En este contexto, la innovación adquiere importancia, no sólo en lo correspondiente a la generación de productos, servicios y procesos que obtengan mejores cuotas de mercado y rentabilidad, sino aquella que aporte o contribuya con el avance de las sociedades mediante innovaciones sociales desde o dentro de la responsabilidad social empresarial (León, Baptista y Contreras, 2012; Solis, et al., 2021).

Las empresas no han creado las características estructurales en las que asoma los diversos problemas sociales, como la pobreza, y los eterniza, por ello su responsabilidad social estará en función de la sociedad que las ha creado, teniendo tres grandes líneas de actuación: Proteger, respetar y remediar (Inglada y Sastre, 2014). Sin embargo, es importante plantearse la siguiente interrogante: ¿La innovación social es un enfoque que puede solucionar los problemas que no ha podido remediar la Responsabilidad social?

La Innovación Social, se presenta como una alternativa de solución frente a estas distintas problemáticas. Uno de los argumentos, según Inglada y Sastre (2014), del porqué los problemas actuales son agobiantes y se han ido esparciendo de manera vertiginosa, es el hecho de que el tejido empresarial se ha formado bajo el sistema capitalista, en el cual se busca como fin supremo el incremento de las utilidades y los beneficios a corto plazo, sin considerar a las personas, la sociedad y el medio ambiente, y se ha aplicado la responsabilidad social empresarial, exclusivamente para asuntos de derechos humanos, integración social y en algunas oportunidades para el cuidado del medio ambiente.

Para Villa (2014), los diferentes y complejos problemas actuales, hacen que los habitantes de cualquier parte del mundo reflexionen si es necesario e importante la reorganización de la manera de gobernar un país, la forma de comportarse de las instituciones y la necesidad de retornar a la educación en valores más humanos, más cívicos y solidarios.

El incremento del intercambio entre las universidades y las partes interesadas, requiere un tipo diferente de compromiso, es lo que se le conoce como la Tercera misión que es la innovación social. Del mismo modo, la labor tradicional de la enseñanza y la investigación, esta misión se focalizaría en la cooperación universitaria al incremento del valor social.

Las universidades, juegan un papel importantísimo como verdaderos especialistas de la producción de conocimiento público, pueden proporcionar no sólo conocimientos específicos sino también actuar como mediadoras entre los diferentes campos de conocimiento, intereses y agendas que figuran en los proyectos comunitarios entre diversos grupos de actores (Bellandi, Donati y Cataneo, 2021).

Según Bellandi, et al. (2021), la participación de las Universidades en la gobernanza de la innovación social es sustancial, puesto que tienen un compromiso público y comunitario dentro de lo que significa los objetivos de esta tercera misión. Para que exista una verdadera gobernanza de la innovación social, libre de conflictos e inconvenientes, la sociedad civil, las empresas, el gobierno y los actores académicos (cuádruple hélice), se propone un marco conceptual de identificación de los nexos comunes, tenencia de estrategias compartidas, aplicación y aprendizaje de reacciones; donde la Universidad es un actor central para comprender los mecanismos de cada fase y con cada integrante de las asociaciones. 
La innovación social a partir de la Universidad, sería una forma de comprender el sentido social del dinamismo universitario en favor del desarrollo personal, cívicosocial, político y cultural de las personas, las comunidades y los pueblos. Está ligada con la oportuna competencia universitaria, arraigada en su misión y visión (Hernández, et al., 2018).

Se refiere también, que las universidades deben centralizar sus esfuerzos en el desarrollo de conocimientos y relaciones con proyectos de innovación social, que fortalezcan los valores, normas, principios de integración, evolución y desarrollo de nuevas relaciones en el contexto social (Hernández, et al., 2018).

El papel de la Universidad en el desarrollo económico es muy relevante y clave, no sólo en la transferencia de conocimiento, sino como una labor con enfoque comunitario general, y no hay duda de que dicha perspectiva está cobrando mucha fuerza en diferentes universidades de Latinoamérica y el Mundo.

Según Fossatti y Batista (2020), en Panamá no se fomenta de forma efectiva la cooperación entre Universidad y Empresa, se percibe falta de estrategias y acuerdos de colaboración para atacar los problemas sociales, se propone impulsar el desarrollo de una economía basada en la innovación, donde las empresas y la Universidad desarrollen investigación, desarrollo e innovación social.

Por ello, la presente investigación tiene como objetivo central dilucidar y entender la complejidad de la definición de la innovación social y el rol que esta, estaría forjándose, desde la Universidad. Para ello, se desarrollan tres puntos clave, la responsabilidad social desde un enfoque crítico, como un camino hacia la innovación social, con dicha base se desarrollará en segundo lugar la perspectiva de la innovación social, clarificando el verdadero significado de esta, desde diferentes enfoques y perspectivas conceptuales de variados autores y contextos. Con dicho recorrido conceptual y teórico, se presentará el tercer punto: La innovación social desde la Universidad, el papel que debe ejercer esta última, como actor clave de la sociedad; para finalmente, llegar a conclusiones contundentes sobre el tema abordado

\section{La responsabilidad social: Enfoque crítico y un camino hacia la innovación social}

Según Colpas, Taron y Fuentes (2019), refieren que se está en un periodo en el cual todas las acciones realizadas tienen una consecuencia, sea esta directa o indirecta, con mayor incidencia en el ámbito de la actividad económica y empresarial en la que se genera una serie de efectos colaterales, que no llegan a ser las mejores para la gran mayoría.

Por esto, urge obtener una mejor relación entre los objetivos de crecimiento económico y de progreso social, aunado a una actitud constante de cuidado, respeto y protección del medio ambiente, es aquí, donde entra a tallar el desarrollo sustentable brindando la visión de una sociedad más próspera y justa en la cual se promueva un ambiente más limpio, seguro y sano para la comunidad.

Para el Banco Interamericano de Desarrollo (BID, 2015), el sector privado cumple un rol fundamental al momento de dar respuestas a los desafíos sociales, puesto que en el sector público no se encuentra todo el conocimiento necesario para determinar cuáles son las principales áreas de intervención y, por tanto, no necesariamente sabe cómo diseñar políticas acordes, por la carencia de recursos o de gestión eficiente.

La cultura y los estilos de vida actuales son considerados insostenibles, y se busca modificarlos hacia alternativas que compatibilicen con la calidad de vida y la conservación ambiental. En la actualidad, el medio ambiente se halla asistido por una gran cantidad de desechos no deseados, debido a que el modelo actual de desarrollo exige grandes cantidades de recursos materiales que no se controlan y que se utilizan irracionalmente, expresándose en una serie de componentes no deseables que terminan contaminando al ambiente (Colpas, et al., 2019).

Aportar hacia un desarrollo sostenible, es el objetivo de la responsabilidad social. Las 
organizaciones, se encuentran sujetas a través de sus diferentes partes interesadas a mayor escrutinio, por este motivo, el bienestar de la sociedad y el medio ambiente se han convertido en el compromiso de una organización.

Al respecto, Guillén, et al. (2020), refieren que con el desarrollo sostenible se busca recapacitar y reacomodar la manera en que los seres humanos deben vivir en el planeta, cuidar los recursos naturales y sobre todo los no renovables para enfrentar el futuro, fortaleciendo a las nuevas generaciones.

Sánchez-González, Gil-Saura y RuizMolina (2020), indican que el desarrollo sostenible se define como la satisfacción de las necesidades de la actual generación, sin afectar la oportunidad de las futuras generaciones. Las empresas deben realizar acciones permanentes para reducir el impacto de sus actividades en el medio ambiente y en la sociedad en general, llegando a construir desarrollo sostenible.

Para Ormaza, et al. (2020) la Responsabilidad Social (RS), es un modelo que puede ser perfectamente aplicable a todo tipo de organización, sin mediar actividades o países, que busca valorar y confrontar su aporte al desarrollo sostenible. Por todo ello, toda organización debe tener como parte de sus objetivos estratégicos y fundamentales la gestión de la RS, así podrán dar respuesta efectiva a los entornos en donde operan.

Las organizaciones, tienen como reto la satisfacción combinada o fusionada entre la sociedad y ellas, se trata pues de promover el desarrollo personal y el bienestar común, lográndose materializar con la satisfacción de las necesidades sociales de los miembros de estas organizaciones como de la comunidad en general, a esto se le llama acciones de responsabilidad social, y hay un interés fuerte en que las organizaciones, independientemente de sus particularidades, desarrollen programas que tengan impacto primero en sus áreas, y luego en cada uno de los miembros de sus grupos de interés (Mendoza, Jaramillo y López, 2020).

¿Pero qué es responsabilidad social empresarial? Arévalo y Moncada (2016), refieren que la Responsabilidad Social
Empresarial (RSE) surge como un concepto para dar solución a los diferentes problemas sociales y ambientales de la actualidad, el cuál fue evolucionando desde lo que se denominó filantropía empresarial.

El comportamiento de una organización en materia de responsabilidad social puede influir, entre otras cosas, en la ventaja competitiva, reputación, la capacidad de atraer y retener trabajadores o miembros, clientes y usuarios, el mantenimiento de la moral, el compromiso y la productividad de los empleados, la percepción de los inversores, propietarios, donantes, patrocinadores y la comunidad financiera, relaciones con empresas, gobiernos, medios de comunicación, proveedores, pares, clientes y la comunidad en la que opera.

León, et al. (2012), indican que la RSE busca lograr la protección del entorno natural y contribuir a mejorar la calidad de vida de los empleados, sus familias, los clientes y demás grupos, para lograrlo concibe dos dimensiones, una interna, que aborda la gestión del recurso humano, la salud, y la seguridad del puesto de trabajo, la gestión del impacto ambiental $\mathrm{y}$ de los recursos naturales; y una externa, concerniente a las comunidades locales, los socios comerciales, los proveedores y los consumidores, derechos humanos, problemas ecológicos y desarrollo sostenible.

Como se pudo denotar de las anteriores aseveraciones, la RSE tiene un enfoque que se puede calificar como reactivo, es decir, de mitigación frente a los daños causados por la operatividad de cualquier organización. La RSE es una herramienta por tanto que busca disminuir el impacto negativo que conllevan sus acciones, en un mundo globalizado con tendencias consumistas devastadoras.

Sin embargo, se puede resaltar la contribución de grandes empresas en América Latina, que han beneficiado a muchas comunidades con programas y estrategias sociales, considerando a la RSE como una estrategia para lograr una ventaja competitiva, siempre y cuando se llegue al objetivo de beneficiar a toda la sociedad, pero lamentablemente, existen casos empresariales 
en los que se implementa la RSE para beneficios individuales, es decir, se realiza con fines de mejorar su imagen corporativa o por hacer parecer que son actores proactivos frente a su realidad. Por ello, América Latina se encuentra rezagada en estos abordajes, y es ahí donde despierta la crítica hacia este modelo, dando el punto de partida hacia otros enfoques más integrales.

Para evitarse una serie de problemas, sobre todo financieros, extremadamente controversiales, se afirma que las organizaciones que actúan bajo la RSE actúan pensando en ganar corazones y mentes, sobre todo de aquellos líderes de la opinión pública. Con el crecimiento de la noción de la RSE, el debate gira en torno a los roles y motivaciones de las empresas cuando llevan acciones fuera del ámbito comercial, se coloca en duda los logros sociales y económicos de esas acciones. Se abre camino al escepticismo y la crítica de las verdaderas intenciones de estas empresas (Alves, Reficco y Arroyo, 2014).

Cabe la reflexión de si la RSE es suficiente como solución efectiva, puesto que se debe comprender que tiene ciertas limitaciones en su contexto y funcionamiento, comenzando por que su desarrollo se da de una manera voluntaria y no prioritaria, lo que genera que muchos empresarios no se involucren en esta gestión de forma preponderante. Así se establece en la ISO 26000 (Chaloemvivatkit y Jaikengkit, 2020), que es la guía de responsabilidad social y busca promover un entendimiento común de la misma, sin embargo, esta no es una norma de sistema de gestión, no contiene requisitos $\mathrm{y}$, como tal, no puede utilizarse para la certificación.

Por otra parte, es claro que el modelo de gestión empresarial actual se ha construido bajo un esquema capitalista donde aparecen con énfasis los conceptos de disminución de costes, acrecentar las ventas, mayor utilidad, entre otras, y han dejado de lado la parte social y ambiental, y son muy pocos los involucrados en esta tarea; por ello, se puede afirmar que nada certifica que las generaciones venideras lleven consigo esta misma ideología, denotando que la RSE podría no ejercerse como debería. ¿Realmente existe conciencia social?

A todo lo anteriormente comentado, Arévalo y Moncada (2016) manifiestan que la RSE se centra sobre todo en minimizar su impacto negativo, no en incluir la generación de valor social positivo como parte medular de sus acciones. Es así que surge una nueva necesidad de abordaje, y se tiene que plantear con el objetivo claro y contundente de instaurar un valor social positivo desde la organización para una sociedad sostenible, sin dejar de lado la concepción de que cualquier organización busque y logre un beneficio económico. ¿Será la innovación social, el enfoque que se requiere? ¿Es el enfoque "proactivo", impulsador de cambios reales y perdurables, que se necesita?

\section{Perspectiva de la innovación social: Un recorrido conceptual}

Por más que la innovación social sea un tema de creciente relevancia en el ámbito académico actual, aún no se ha logrado una definición que sea aprobada por la gran mayoría de los intelectuales de la materia. Definitivamente, el concepto de innovación social aún debe ser desarrollado a profundidad para lograr una idea que englobe todo lo concebido por este término. Hoy en día, las definiciones del término en cuestión se basan meramente en el sector en el que se desarrolla, ignorando sus características inherentes y elementos diferenciales, pero para entender realmente que es la innovación social, esta no se puede desprender del contexto cultural del cual emerge (Hernández, Tirado y Ariza, 2016).

Habiendo dicho esto, se ha realizado un exhaustivo proceso de revisión, del cual se pudo extraer definiciones relevantes de lo que es la innovación social. Al momento de realizar la selección de los conceptos se utilizaron criterios de citación objetivos y relevantes. Para obtener estas definiciones, se efectuó una revisión bibliográfica a través de las 
principales bases de datos científicas, se utilizó como palabra clave innovación social en el idioma inglés y español por ser los ámbitos de mayor incidencia. A raíz de esto, se obtuvieron un total de 22 definiciones destacables que serán analizadas de forma crítica después de su presentación en el Cuadro 1.

\section{Cuadro 1 \\ Perspectivas del concepto de innovación social}

\section{Autor (es)}

Westley y Antadze (2010)

León, et al. (2012)

Khutrakun (2013)

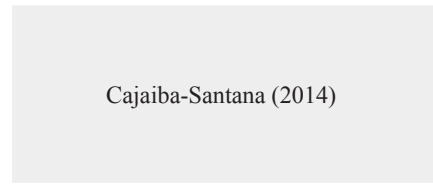

O’Byrne, et al. (2014)

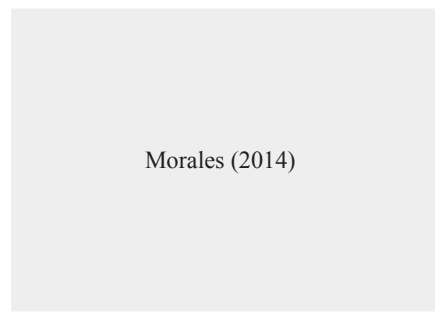

Conrad (2015)

Tracey y Stott (2016)

Bittencourt y De Abreu (2016)

\section{Perspectivas diversas del concepto de innovación social}

La innovación social es un proceso complicado de introducción de nuevos productos, procesos o programas que modifican profundamente las rutinas básicas, los recursos y los flujos de autoridad o las creencias del sistema social en el que se desarrolla la innovación, aquellas innovaciones sociales exitosas tienen durabilidad y amplio impacto.

Una innovación social puede ser un producto, proceso de producción o la tecnología, como la innovación en general. También puede ser un principio, una idea, una intervención o alguna combinación de ellos.

La innovación social es un conjunto de actividades nuevas y socialmente valiosas, que son iniciadas e implementadas por personas que viven en esa área en particular.

La innovación social toma forma cuando se establece una nueva idea, una forma diferente de pensar y de actuar que cambia los paradigmas. Las innovaciones sociales son nuevas prácticas sociales creadas a partir de acciones colectivas, intencionales y orientadas a la consecución de objetivos, dirigidas al cambio social a través de la reconfiguración de cómo los objetivos sociales se logran.

La innovación social se puede comprender como la implementación exitosa de actividades, como ideas, prácticas u objetos, gracias a colaboraciones y asociaciones, de manera que impacten positivamente a la sociedad al mejorar la prestación de servicios públicos.

Considera tres elementos base de la innovación social: a) nuevas ideas, b) necesidades sociales; c) cambio social (nuevas relaciones); refiriendo que es el tercero el que puede ser de mayor ayuda para discernir que experiencias pueden ser consideradas como tal. Políticas o acciones que tienen como meta mejorar la situación de un grupo social en desventaja o aliviar ciertas carencias son buenas para la sociedad, pero a menos que la participación de los beneficiados sea activa, que haya un proceso de reflexión y cambios en las relaciones, no puede considerarse como innovación social. En este sentido, los términos relacionados: empresa social, economía social, emprendedor social, se conjugan con el de innovación, y las políticas públicas contienen una dimensión de innovación social cuando estos cambios ocurren.

La innovación social es entendida como la intención de lograr un cambio social para hacer que las cosas sucedan en un mundo complejo.

Innovación social es un término muy discutido, suele ser definido de manera general como la creación e implementación de nuevas soluciones a problemas que ocurren en la sociedad.

Refieren que la innovación social se define como un conjunto de ideas novedosas (por ejemplo, productos, servicios o modelos de acción) que satisfacen las necesidades humanas y suscitan nuevas relaciones sociales, no solo para beneficiar a la sociedad sino también con el fin de mejorar su capacidad de actuar. 


\section{Cont... Cuadro 1}

Bitencourt, et al. (2016)

Phillips, Alexander y Lee (2017)

Melamed-Varela, Blanco-Ariza, y Rodríguez-Calderón (2018)

Ahmed, Ashikuzzaman y Mahmud (2017)

Unceta, Castro-Spila y García (2017)

Alves, De Souza y Da Silva (2018)

Silva y Pedroza (2018)

Anechitei (2018)
La innovación social es definida como la aparición de nuevos arreglos sociales, organizativos y disposiciones institucionales o nuevos productos y servicios diseñados para afrontar las aspiraciones, satisfacer las necesidades o lograr la solución a consecuencia de un reto social.

Los autores de acuerdo con los postulados de la OCDE en 2011, manifiestan que la importancia de la innovación social se coloca de manifiesto en el cómo dar respuesta a problemas y necesidades sociales no resueltas o insuficientemente atendidos, y que el gobierno o el mercado comercial las dirigen sin éxito, como identificar y suministrar nuevos productos, servicios que mejoran la calidad de vida de las personas y comunidades; identificando e implementando nueva mano de obra, procesos de integración del mercado, nuevas competencias, puestos de trabajo, así como nuevas formas de participación en el lugar de trabajo.

La innovación social como el proceso orientado a la búsqueda, diseño e implementación de alternativas que respondan a las problemáticas sociales de forma novedosa y contribuyente al desarrollo socioeconómico en los territorios, distinguiéndose por su originalidad, pertinencia y transformadora de escenarios e ideas. Su despliegue del concepto implica dimensionar factores como: el cambio frontal, los principios de gobernanza y los procesos que orientan las estrategias operativas, siendo estos determinantes en el alcance y la propuesta de valor que oferta apropiar la innovación social.

Definen la innovación social como la creación y el uso de nuevas ideas sobre las personas y sus interacciones dentro de un sistema social, las cuales también involucran la formación de nuevos métodos y formas de interacción social.

Para llevar a cabo una innovación social, las organizaciones deben poseer al menos tres competencias claves: (a) interpretar un problema social (según una perspectiva sobre las causas, y sus posibles efectos y alternativas); b) asimilar/transformar la interpretación de acuerdo con un patrón interno de conocimiento y experiencia, integrando este conocimiento en prototipos, productos, servicios, métodos que proporcionen respuestas al problema que buscamos. c) explotar los resultados obtenidos, es decir, experimentar y transferir soluciones, y evaluar el impacto.

Se refiere que en general, la innovación social es una nueva respuesta a una situación social insatisfactoria. La innovación social sostiene esto, porque se refiere al bienestar de las personas y/o comunidades, definiéndose a sí mismo como una acción o cambio duradero destinado a desarrollar a las personas, los territorios o las personas, y negocios. La innovación social no asume una forma particular, sino que puede ser de procedimiento, organizacional o institucional; y van más allá de las tendencias temporales, y con efectos que influyen en el desarrollo futuro de la sociedad.

La innovacion social se caracteriza principalmente por la colaboración de diversos actores, lo cual hace posible la obtención de un potencial con valor más grande del capital social o región con el objetivo de satisfacer las necesidades humanas y la generación de relaciones entre individuos y grupos de diferentes espacios y escalas.

La innovación social es una nueva solución estructural a un problema social, una que produce un cambio social en el sentido de una mejora de la calidad de vida de las personas. En general, la innovación social es una "nueva respuesta" a un problema social, situación considerada insatisfactoria, situación que puede manifestarse en todos los sectores de la economía y de la sociedad civil. La innovación social responde a esto porque se ocupa del bienestar de las personas, individuos y/o comunidades. 


\section{Cont... Cuadro 1}

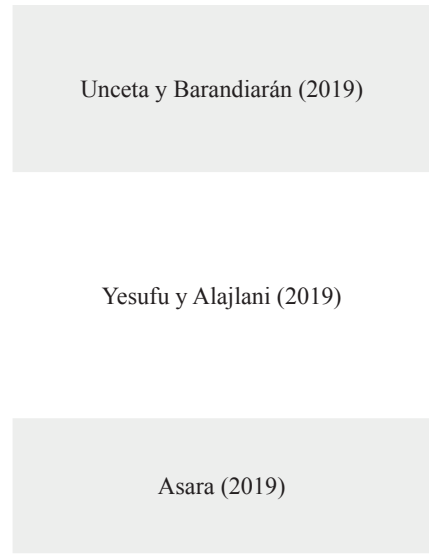

Sampedro-Palacios y Pérez-Villar (2019)

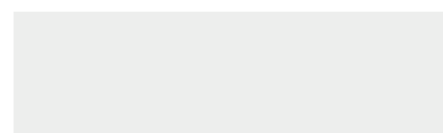

Domínguez (2019)
La innovación social se puede entender como el resultado de nuevos modelos de gobierno para facilitar la atención de problemas y necesidades de la población que no están cubiertas. La innovación social puede afectar e influir en las formas en que diseñamos y abordamos un problema social; por tanto, aborda la regeneración de las facetas públicas, las políticas públicas, gestión pública, la buena gobernanza y la política.

Se aplica el término "innovación social" a la transformación de la sociedad a través de la creación de nuevos productos, servicios, gestión, emprendimiento social, desarrollo del gobierno y del capital humano. Por consiguiente, se está frente a nuevos productos, servicios, modelos, mercados, procesos, etc. que satisfacen simultáneamente una necesidad social de manera más eficaz que las soluciones existentes y generan capacidades nuevas o mejoradas y relaciones y/o un mejor uso de los activos y recursos. Por lo tanto, las innovaciones sociales son buenas para la sociedad y aumentan su capacidad de acción.

La innovación social puede ser entendida como un grupo de procesos cooperativos y prácticos que invitan a participar a los ciudadanos, resultando en la creación de servicios públicos que respondan a necesidades sociales materiales o inmateriales.

La innovación social aplica como herramienta en la cual se impulsa la adquisición de una sociedad accesible e inclusiva a través de la creación y modificación de productos y servicios con la finalidad de mejorar la vida de las personas, así como su autonomía.

El marco de referencia de la innovación social, se encuentra en la solución de problemas sociales a partir de nuevas relaciones o colaboraciones entre diferentes actores, en los que se incluye la universidad; además, independiente de si estos actores se dedican a la creación de políticas públicas (Estado), generación de rentabilidad (empresas), atención y cooperación para el desarrollo (ONG) o a la organización comunitaria (las organizaciones de base), todos concuerdan en que las innovaciones sociales abordan las necesidades a través del conocimiento y la generación de nuevas soluciones tecnológicas, para mejorar las prácticas sociales u organizativas; colaboran con la reducción de las desigualdades; y se centran en mejorar la calidad de vida.

Fuente: Elaboración propia, 2021.

La innovación social suele ser presentada como un instrumento normativo que busca resolver problemas en el ámbito social mediante la creación de nuevos servicios o nuevos productos. Sin embargo, aquella definición limita incorrectamente el campo de acción de la innovación social. Primero, porque una respuesta a un problema social no es necesariamente una innovación social, puesto que incluso las innovaciones técnicas o ingenieriles podrían resolver problemas sociales. Segundo, porque propone la implicancia de algo tangible, es decir, la creación de un producto o servicio, lo que es incoherente con la inmaterialidad, puesto que las innovaciones sociales se manifiestan también en cambios de actitudes, comportamiento, o percepciones, que dan lugar a nuevas prácticas sociales.

En tercer lugar, el aspecto social de la innovación se relaciona a un cambio social y este debe ser la característica principal puesta en evidencia, recalcando que no se está sólo frente a cambios en la forma de actuar de los agentes sociales y sus interacciones, sino también se habla de los cambios en el entorno 
social. Es decir, el contexto en el que se llevan a cabo estas acciones a través de la creación de nuevas instituciones y nuevos sistemas sociales.

La innovación social toma forma cuando se establece una nueva idea, una forma diferente de pensar y de actuar que cambia los paradigmas. Las innovaciones sociales son nuevas prácticas creadas a partir de acciones colectivas, intencionales y orientadas a la consecución de objetivos, dirigidas al cambio social a través de la reconfiguración de cómo los objetivos sociales se logran.

La mayoría de las investigaciones sobre el tema están vinculadas al empresariado con visión social o a iniciativas de política pública, que no consideran otros factores de la innovación social. Sin embargo, CajaibaSantana (2014), presenta un espectro más amplio de fuentes de innovación social, reconociendo que estas pueden asumir su forma y ser difundidas a través del mercado (como nuevos servicios, modelos de negocio, logística y conceptos de aplicación), así como también, con factores tecnológicos y de infraestructura, redes sociales y movimientos sociales, además, a través de directrices y apoyo gubernamentales, entre otros tantos factores variables.

La innovación social puede brindar diferentes tipos de resultados. Verbigracia, puede tomar la forma de nuevas instituciones, movimientos sociales, nuevas prácticas sociales, o diferentes estructuras de colaboración, pero independientemente de la fuente de la innovación social, los conceptos de acción social y cambio social, son fundamentales para su proceso.

Según Cajaiba-Santana (2014), se necesita una visión más holística del fenómeno de la innovación social, puesto que esta se ve influenciada tanto por los agentes como por las estructuras sociales, refiriendo que la innovación social se basa en acciones colectivas que tienen lugar dentro de un sistema social determinado, y que se establecen por las instituciones compartidas, pero también por la historia y la cultura, es decir, el contexto (histórico y cultural) en el que se produce la innovación social es un factor muy importante. Como se observa, los académicos coinciden en la mayor parte de los aspectos que involucran una innovación social, demostrando que existe un consenso en cuanto a la atención de las necesidades sociales y ambientales no cubiertas por gobierno o el mercado y su contribución a mejorar la calidad de vida de los grupos más vulnerables. Se trata de innovaciones que solucionan problemas sociales de forma novedosa, eficaz, eficiente, sostenible, participativa e incluso colaborativa. Sin embargo, también se observan en estos enfoques posturas opuestas. En un extremo, se encuentran aquellos que consideran que sólo se puede llamar innovación social a aquella idea que plantea únicamente beneficios para la sociedad y excluye tajantemente un beneficio económico para la compañía. En el otro, están las posturas que plantean la generación de valor económico de manera bilateral, es decir, tanto para los productores como para la sociedad. En medio, se encuentran los intelectuales que perciben a la innovación social como un proceso evolutivo o que simplemente creen que todo problema social generará un valor económico.

En esencia, resaltan como aspectos reiterativos en estos enfoques, la atención a las necesidades sociales de determinados grupos vulnerables, que no son eficaz ni eficientemente atendidos por gobiernos o el mercado. Implica la sinergia que puede producirse entre el sector público, el privado y $\sin$ fines de lucro. El escenario ideal, es producir cambios en valores, actitudes y procesos, entre otros, que contribuyan a un mayor bienestar de individuos, comunidades, consumidores y productores.

Melamed-Varela, et al. (2018), refieren que se debe catalogar a la innovación social en niveles, de acuerdo a los objetivos y actores vinculados. Así en el nivel incremental, el objetivo es mejorar las carencias o incompetencias a través de productos y servicios. Mientras que, a nivel institucional se busca reorganizar las estructuras enfatizando esfuerzos en los mercados y comunidades. Finalmente, la innovación social disruptiva 
se desarrolla con el objetivo de transformar paradigmas, especialmente aquellos que involucran políticas públicas y movimientos sociales.

\section{La innovación social desde la Universidad}

Según Salgado y Patera (2017), el motivo de ser de una universidad implica toda una trayectoria de exploración dónde la verdad prima en la producción del conocimiento, considerando también la licitud científica, la autenticidad sociocultural y la disposición de poder comunicarse de una forma integrada sin apartar a la persona como objeto, núcleo y razón de esta práctica.

La Universidad, en ese sentido, se enfrenta ante un reto intenso de tener el compromiso de instruir o preparar a estudiantes y catedráticos, generando de esta forma, una ciudadanía crítica (base del juicio moral) y con iniciativa de producir cambios aplicando la sensibilidad antropológica. Frente a ello, se condiciona la siguiente interrogante: $\mathrm{Si}$ el conocimiento debiera ser el principal modelo de sociedades, por consiguiente, ¿en qué medida la Universidad está ejecutando el conocimiento y no sólo tener un enfoque informativo? Así mismo, si la «sociedad del conocimiento» compromete un crecimiento y no implica de otros medios del poder o del control, sino más bien que tenga una validación propia, ¿qué modelo de conocimiento debe desarrollar la Universidad?

Las instituciones de educación superior, están enfrentándose a nuevos desafíos, los cuales generan en estas reformular su rol en relación a la creación, gestión y divulgación de los nuevos conocimientos, de manera que las tareas de investigación y de extensión, al igual que la transferencia, deben estar debidamente vinculadas con la docencia, donde ambas partes puedan perfeccionarse mutuamente. De acuerdo a lo planteando y de esa forma, la Universidad puede cumplir las funciones que la nueva relación educación superior- sociedad le exige (Fernández, 2014).
Ya se ha sugerido que las universidades o institutos consideren los cambios respectivos en su política de educación, de manera que contribuyan al mejoramiento de la gobernabilidad y sobre todo para un beneficio mayor de democracia en la sociedad, donde puedan plantearse estrategias a fin de mejorar y disminuir la injusticia que se vive en esta sociedad, donde se evidencia desigualdad en relación a la pobreza y marginalidad.

Dentro de un Estado Social y Democrático, la Universidad debe ser canalizada a la prestación de un servicio público de generación de conocimiento donde los intereses generales de la ciudadanía, defender el bien común, funcionando como un agente de cambio y mejora social deben ser considerados, ¿será que la cultura empresarial está desorientando esa función natural con la creación de universidades privadas?

Existen muchas necesidades sociales, motivo por el cual la Universidad debe cubrirlas priorizando así no sólo las demandas del mercado si no también su propia función social, de manera que pueda consolidar un acuerdo en conjunto con la sociedad. No sólo que se considere como un ente académico si no que esta actúe dinámicamente para transformar la sociedad. En consecuencia, debe tener como base la enfatización en el compromiso y responsabilidad social y la conexión con sectores menos privilegiados (Díez, 2018).

Martínez (2015) sostiene que, al hablar de innovación social, es mencionar que existe todo un conjunto organizacional donde intervienen diferentes actores con el fin de desarrollar e impulsar un desenlace ante los retos del entorno social y global. Son manifestaciones y expresiones creativas (no sólo empresariales), que indagan cómo reducir la pobreza y desigualdad social del país, de ahí la importancia de la Universidad como socio activo estratégico.

Asimismo, Villa (2014) señala que, para enfatizar la innovación social en la Universidad, se deben considerar seis dimensiones: La primera, hace referencia a un diseño curricular con enfoque social; la segunda, que el sistema organizacional sea respuesta ante los procesos 
y fines de dicha innovación social; la tercera, que la investigación, el desarrollo e innovación sea entendida como una mejora para la sociedad; la cuarta, aceptar y ser partícipe de una responsabilidad socio-ecológica, teniendo como base el desarrollo de un pensamiento crítico y la práctica responsable de ella misma; la quinta, que la Universidad como tal debe ser un referente y figura social, desarrollando proyectos y programas con el interés de mejorar la calidad de vida de la sociedad; y la sexta, la preparación de profesionales con liderazgo y competitividad en diferentes niveles a fin de colaborar con otras instituciones y zonas.

\section{Conclusiones}

Existen una serie de problemas sociales $\mathrm{y}$ ambientales que evolucionan a gran escala, en un contexto donde los gobiernos actuales no cubren en su integridad dichos fenómenos (por diferentes situaciones que no es materia de este artículo, pero que da para su tratamiento en un futuro), percibiéndose la necesidad de recurrir a la RSE, pero se ha visto que no resulta suficiente, debido a que no se garantiza una ayuda invariable a las problemáticas latentes, su enfoque es reactivo y de mitigación, más que de acciones innovadoras de largo alcance.

Todo esto, se da también en un escenario donde la gestión de las empresas no se condice con la misión y visión de las mismas, buscando sólo un incremento de su reputación con acciones paliativas, que más parecen filantrópicas básicas, que la contribución a un desarrollo óptimo y sostenible, desde lo económico, social y ambiental.

Se puede concluir, que el término de innovación social es difuso, ambiguo y multivariado, aun así, existen intentos de llevar a cabo sistematizaciones de las diferentes definiciones con el fin de establecer los elementos, características, rasgos o variables comunes al fenómeno de la innovación social. Lo que está quedando claro, que existe un consenso en cuanto a la atención de las necesidades sociales y ambientales no cubiertas por un gobierno o por el mercado y también su contribución a mejorar la calidad de vida de los grupos más vulnerables, desde un enfoque técnico y aplicado, fundamentado en los recursos y capacidades necesarios para la práctica social, con un abordaje de soluciones proactivo y de largo plazo.

Se trata entonces de factores innovadores, que producen mejoras perdurables ante las inefectividades del mercado, una reconfiguración de los modelos y estructuras de gestión existentes, y la ruptura de paradigmas nada conducentes a la mejora de la calidad de vida.

Existe una demanda constante para que la Universidad se oriente hacia la sostenibilidad y el desarrollo sostenible. Se le exige su participación y el asumir un papel integral en la vida social, cultural, política, y en los aspectos económicos de la sociedad, con soluciones innovadoras desde su papel central, que es la investigación, pero con rostro social, para así lograr una transformación realmente significativa, urgente y necesaria.

Como futura línea de investigación, se debe abordar este tema de indagación con un método empírico, para descubrir las implicancias de la innovación social en la gestión universitaria, pretendiendo confirmar la intuición del autor acerca de los efectos positivos de la innovación social sobre dicha gestión. En caso de respuesta positiva, identificar cuáles son concretamente estos factores determinantes que condicionan la innovación social, sería el segundo paso imprescindible. Finalmente, elaborar un mapa de estos factores para hacer emerger un modelo teórico que permita categorizarlas y servir de marco metodológico y práctico en las universidades del Perú. Todo este abordaje se debe dar mediante un análisis explicativo, con el uso de la estadística inferencial, específicamente un modelo de ecuaciones estructurales.

\section{Referencias bibliográficas}

Abreu, J. L. (2011). Innovación social: 
Conceptos y etapas, Daena, International Journal of Good Conscience, 6(2), 134-148.

Ahmed, J. U., Ashikuzzaman, N. M., y Mahmud, A. S. M. (2017). Social innovation in education: BRAC boat schools in Bangladesh. Journal of Global Entrepreneurship Research, 7(20). http://doi.org/10.1186/s40497017-0077-z

Alves, A. C. A., De Souza, B., y Da Silva, J. C. L. (2018). Social innovation and the promotion of local economic development. Innovation \& Management Review, 16(1), 55-71. http://doi.org/10.1108/INMR-10$\underline{2018-0074}$

Alves, M. A., Reficco, E., y Arroyo, J. (2014). Perspectivas sobre la situación $\mathrm{y}$ proyección de la responsabilidad social empresarial en américa latina. RAE Revista de Administração de Empresas, 54(1), 10-11. https://doi. org/10.1590/S0034-759020140102

Anechitei, A-A. (2018). Social innovation through urban regeneration - a local model. Review of International Comparative Management, 19(3), 244-251. https://doi.org/10.24818/ $\underline{\text { RMCI.2018.3.244 }}$

Arévalo, Y. P., y Moncada, D. A. (2016). La innovación social como un paso adelante de la responsabilidad social empresarial generalidades, una visión de Bogotá (Tesis de pregrado). Universidad de La Salle, Bogotá, Colombia.

Asara, V. (2019). The redefinition and coproduction of public services by urban movements: The Can Batlló social innovation in Barcelona. Partecipazione e Conflitto, 12(2), 539-565. $\quad$ https://doi.org/10.1285/ i20356609v12i2p539

Banco Interamericano de Desarrollo -
BID (2015). Innovación social empresarial: Impulsando el emprendedurismo para el desarrollo social. II Cumbre empresarial de las Américas. BID. https://publications. iadb.org/publications/spanish/ document/Innovaci\%C3\%B3nsocial-y-empresarial-Impulsando-elemprendedurismo-para-el-desarrollosocial.pdf

Bellandi, M., Donati, L., y Cataneo, A. (2021). Social innovation governance and the role of universities: Cases of quadruple helix partnerships in Italy. Technological Forecasting and Social Change, 164, 120518. https://doi. org/10.1016/j.techfore.2020.120518

Bittencourt, B. D. L., y De Abreu, L. F. (2016). Social innovation and development policies: the case of Land Exchange (Bolsa de Terras). Revista de Administração Pública, 50(5), 795817. https://doi.org/10.1590/0034$\underline{7612151759}$

Bitencourt, C. C., Bittencourt, D. A., Barin, L., y Raufflet, E. (2016). Introduction to special edition social innovation: researching, defining and theorizing social innovation. RAM, Revista de Administração Mackenzie, 17(6). https://doi.org/10.1590/167869712016/administracao.v17n6p14-19

Cajaiba-Santana, G. (2014). Social innovation: Moving the field forward. A conceptual framework. Technological Forecasting and Social Change, 82(1), 42-51. https://doi.org/10.1016/j. techfore.2013.05.008

Chaloemvivatkit, N., y Jaikengkit, A-O. (2020). Applying ISO 26000 and the sufficiency economy philosophy to measure the sustainability of state owned enterprises in Thailand. International Journal of Innovation and Sustainable Development, 14(3), 330-350. https://doi.org/10.1504/ 


\section{IJISD.2020.108055}

Chambon, J-L., David, A., y Devevey, J-M. (1982). Les innovations sociales. Presses Universitaires de France.

Christensen, C. M, Baumann, H., Ruggles, R., y Sadtler, T. M. (2006). Disruptive innovation for social change. Harvard Business Review, 84(12), 94-101.

Colpas, F., Taron, A., y Fuentes, L. (2019). Innovación social y sostenibilidad en América Latina: Panorama actual. Revista Espacios, 40(1), 1-9.

Comisión Económica para América Latina y el Caribe - CEPAL (2011). Innovación social y desarrollo económico local. CEPAL https://www.cepal.org/es/ publicaciones/6181-innovacionsocial-desarrollo-economico-local

Conejero, E., y Redondo, J. C. (2016). La innovación social desde el ámbito público: Conceptos, experiencias y obstáculos. Gestión y Análisis de Políticas Públicas, Nueva Época, (15), 23-42. https://doi.org/10.24965/gapp. $\underline{\mathrm{v} 0 \mathrm{i} 15.10310}$

Conrad, D. H. (2015). Education and social innovation: The youth uncensored project- a case study of youth participatory research and cultural democracy in action. Canadian Journal of Education, 38(1), 1-25.

Crozier, M., y Friedberg, E. (1993). Die zwänge kollektiven handelns. Über macht und organisation. Frankfurt and Main: Verlag Anton Hain.

Díez, E. J. (2018). Universidad e investigación para el bien común: La función social de la Universidad. Aula Abierta, 47(4), 395-402. $\quad$ https://doi.org/10.17811/ rifie.47.4.2018.395-402

Domínguez, E. M. (2019). Innovación social: Nociones de organismos multilaterales y concepciones universitarias. Revista Virtual Universidad Católica del
Norte, (56), 72-88.

Echevarría, J. (2008). El Manual de Oslo y la innovación social. ARBOR Ciencia, Pensamiento y Cultura, 184(732), 609-618. https://doi.org/10.3989/ arbor.2008.i732.210

Fernández, N. (2014). Universidad, sociedad y conocimiento reflexiones para el debate.Avaliação:RevistadaAvaliação da Educação Superior, 19(3), 663687. https://doi.org/10.1590/S141440772014000300008

Fossatti, A., y Batista, D. (2020). Universitycompany collaboration in panamá: A propossal for the problem solutions in key sectors of the economy. Revista Venezolana de Gerencia, 25(92), 1716-1740. https://doi.org/10.37960/ rvg.v25i92.34291

Guillén, J., Calle, J., Gavidia, A. M., y Vélez, A. G. (2020). Desarrollo sostenible: Desde la mirada de preservación del medio ambiente colombiano. Revista de Ciencias Sociales (Ve), XXVI(4), 293-307. https://doi.org/10.31876/rcs. v26i4.34664

Hernández-Ascanio, J., Tirado-Valencia, P., y Ariza-Montes, A. (2016). El concepto de innovación social: Ámbitos, definiciones y alcances teóricos. CIRIEC-España, Revista de Economía Pública, Social y Cooperativa, (88), 165-199.

Hernandez, P. H., Virviescas, J., Martinez, J. L., y Hernandez, H. (2018). Deontology and university social responsibility: Foundations for social innovation in the post-conflict era. Universidad de la Costa.

Inglada, M. E., y Sastre, J. M. (2014). Reflections on globalization, poverty and development: Impact on social economy organization. REVESCO. Revista de Estudios Cooperativos, (116), 160-179. 
Khutrakun, A. (2013). Process and dynamics of social innovation: Case studies of local initiatives in Northern Thailand. Japan Social Innovation Journal, 3(1), 12-18. https://doi.org/10.12668/ jsij. 3.12

León, M. D. F., Baptista, M. V., y Contreras, H. (2012). La innovación social en el contexto de la responsabilidad social empresarial. Forum Empresarial, 17(1), 31-63. https://doi. org/10.33801/fe.v17i1.3745

Martínez, M. C. (2015). La Innovación Social en la Educación Superior de México. RIDE Revista Iberoamericana para la Investigación y el Desarrollo Educativo, 6(11) 637-653.

Melamed-Varela, E., Blanco-Ariza, A. B., y Rodríguez-Calderón, G. R. (2018). Perspectivas de la innovación social: $\mathrm{Su}$ revisión desde la responsabilidad en la gestión de organizaciones. En A. Beltrán, R. Echeverry, C. Restrepo y A. Rodríguez (Comps.), Investigación en Administración y su impacto en comunidades académicas internacionales (pp. 2742-2759). Fundación Universidad Externado de Colombia.

Mendes, A., Batista, A., Fernandes, L., Macedo, P., Pinto, F., Rebelo, L., Ribeiro, M., Ribeiro, R., Sottomayor, M., Tavares, M., y Verdelho, V. (2012). Barriers to Social Innovation. A deliverable of the project: "The Theoretical, empirical and policy foundations for building social innovation in Europe" (TEPSIE). European Comission. https://www.researchgate.net/ publication/299706323 Barriers to Social Innovation A deliverable of the project The theoretical empirical and policy foundations for building social innovation in Europe TEPSIE

Mendoza, D. L., Jaramillo, M. C., y López, D.
D. (2020). Social responsibility of the university of La Guajira with respect to indigenous communities. Revista de Ciencias Sociales (Ve), XXVI(2), 95-106. https://doi.org/10.31876/rcs. $\underline{\mathrm{v} 26 \mathrm{i} 2.32426}$

Mesthene, E. G. (1970). Technological change: Its impact on man and society. Harvard University Press.

Morales, D. A. (2014). Innovación social y acción colectiva, un estudio de caso: Ecoagricultores del Sur. Revista de Estudios Políticos, (33), 75-95. http://dx.doi.org/10.1016/S0185$\underline{1616(14) 70277-X}$

O’Byrne, L., Miller, M., Douse, C., Venkatesh, R., y Kapucu, N. (2014). Social innovation in the public sector: The case of Seoul metropolitan government. Journal of Economic and Social Studies, 4(1), 53-71. https://doi. org/10.14706/JECOSS11414

Ormaza, J., Ochoa, J., Ramírez, F., y Quevedo, J. (2020). Responsabilidad social empresarial en el Ecuador: Abordaje desde la Agenda 2030. Revista de Ciencias Sociales (Ve), XXVI(3), 175193. https://doi.org/10.31876/rcs. v26i3.33241

Parada, J. E., Ganga, F. A., y Rivera, Y. Y. (2017). Estado del arte de la innovación social: una mirada a la perspectiva de Europa y Latinoamérica. Opción, 33(82), 563-587.

Phillips, W., Alexander, E. A., y Lee, H. (2017). Going it alone won't work! The relational imperative for social innovation in social enterprises. Journal of Business Ethics, 156(2), 315-331. $\quad$ https://doi.org/10.1007/ s10551-017-3608-1

Salgado, J. P., y Patera, S. (2017). Nuevos indicadores de producción del conocimiento en la universidad, desde la impronta de una cultura de 
evaluación propendida a la innovación social. El caso de la Universidad Politécnica Salesiana, de Ecuador. Revista Cubana de Educación Superior, 36(3), 116-136.

Sampedro-Palacios, C. B., y Pérez-Villar, J. (2019). Innovación social como herramienta en la transformación de una sociedad inclusiva. Accesibilidad e innovación social. Prospectiva, (28), 93-119. https://doi.org/10.25100/prts. $\mathrm{v} 0 \mathrm{i} 28.7218$

Sánchez-González, I., Gil-Saura, I., y RuizMolina, M. E. (2020). Efectos del desarrollo sostenible percibido por el consumidor. Una propuesta de modelo de hipermercados en Ecuador. Estudios Gerenciales, 36(154), 27-42. https://doi.org/10.18046/j. estger.2020.154.3470

Silva, M. L., y Pedroza, Á. R. (2018). Social innovation: A social shared competence. Education In The Knowledge Society (EKS), 19(2), 4762.

Solis, J. B., Neira, M. L., Ormaza, J. E., y Quevedo,J.O.(2021).Emprendimiento e innovación: Dimensiones para el estudio de las MiPymes de AzoguesEcuador. Revista de Ciencias Sociales (Ve), XXVII(1), 315-333. https://doi. org/10.31876/rcs.v27i1.35318

Tracey, P., y Stott, N. (2016). Social innovation: A window on alternative ways of organizing and innovating. Innovation: Organization \& Management, 19(1), 51-60. https://doi.org/10.1080/144793 $\underline{38.2016 .1268924}$

Unceta, A., y Barandiarán, X. (2019). Social innovation as an instrument for public innovation. Universia Business Review, 61(1), 100-111.

Unceta, A., Castro-Spila, J., y García, J. (2017). The three governances in social innovation. Innovation: The European Journal of Social Sciences, 30(4), 406420. https://doi.org/10.1080/13511610 .2017 .1279537

Villa, A. (2014). La innovación social en el ámbito universitario: una propuesta para su diagnóstico y desarrollo. RAES. Revista Argentina de Educación Superior, 6(8), 188-218.

Westley, F., y Antadze, N. (2010). Making a difference: Strategies for scaling social innovation for greater impact. The Innovation Journal: The Public Sector Innovation Journal, 15(2), article 2.

Yesufu, L., y Alajlani, S. (2019). Measuring social innovation for education and resource development in refugee camps: A conceptual study. International Journal of Higher Education, 8(4), 208-220. https://doi. org/10.5430/ijhe.v8n4p208 